\title{
ON STRONG BOUNDS FOR SUMS OF INDEPENDENT RANDOM VARIABLES WHICH TEND TO A STABLE DISTRIBUTION( $\left.{ }^{1}\right)$
}

\author{
BY \\ MIRIAM LIPSCHUTZ
}

1. Notation and generalities. We shall in this paper deal with sums of independent, identically distributed, positive, continuous random variables, whose distribution lies within the domain of attraction of a stable law. We denote the random variables by $X_{k}, k=1,2, \cdots$, their distribution function by $F(x)$, and their sum by $S_{n}=\sum_{k=1}^{n} X_{k}$. We thus are given

$$
P\left(X_{k} \geqq x\right)=1-F(x)=h(x) / x^{\gamma} \quad \text { for } x>x_{0}
$$

where $0<\gamma<2$, and $h(x)$ is such that for any positive constant $c$,

$$
\lim _{n \rightarrow \infty} h(c n) / h(n)=1 \text {. }
$$

For $1<\gamma<2$ we write $\mu=E(X)$ and $W_{n}=S_{n}-n \mu$. We define $b_{n}$ so that

$$
1-F\left(b_{n}\right)=1 / n \text {. }
$$

The distribution function $G_{\gamma}(x)$ is to denote the stable law of index $(\gamma,-1)$; its characteristic function is given by

$$
\begin{aligned}
\phi_{\gamma}(t) & =\exp \left[-|t| \gamma\left(\cos \frac{\gamma \pi}{2}-i \sin \frac{\gamma \pi}{2} \operatorname{sign} t\right) \Gamma(1-\gamma)\right] & & (0<\gamma<1) \\
& =\exp \left[|t| \gamma\left(\cos \frac{\gamma \pi}{2}-i \sin \frac{\gamma \pi}{2} \operatorname{sign} t\right) \gamma \Gamma(-\gamma)\right] & & (1<\gamma<2) .
\end{aligned}
$$

It is known [1] that when (1) is satisfied by the random variables $X_{k}$ then

$$
\begin{array}{ll}
\lim _{n \rightarrow \infty} P\left(S_{n}<b_{n} x\right)=G_{\gamma}(x) & \text { for } 0<\gamma<1, \\
\lim _{n \rightarrow \infty} P\left(W_{n}<b_{n} x\right)=G_{\gamma}(x) & \text { for } 1<\gamma<2 .
\end{array}
$$

Throughout the paper the symbols $C, c, C_{1}, M_{1}$, etc. will stand for constants

Presented to the Society, September 5, 1952 under the title Strong laws for sums of independent random variables with stable distributions; received by the editors April 29, 1955.

( ${ }^{1}$ This work was partially sponsored by the Office of Scientific Research under Air Force Contract AF 18(600)-442, Project R-345-20-7. 
which are not necessarily the same each time the symbol is used. Likewise for $\epsilon, \eta, \delta$, etc. which denote arbitrarily small given positive numbers.

The function $h(x)$ appearing in (1) determines the order of magnitude of the error [2] in the limit theorems (4). In what follows we shall make the following assumptions on the function $h(x)\left({ }^{2}\right)$ : a. Let $r(n)$ be a function which tends to infinity with $n$ and

$$
(\lg n)^{1-\delta}=O(r(n)) \text { for any } \delta>0 .
$$

b. Assume that for

$$
r(n)^{-2} \leqq x<r(n)^{3 / \gamma}
$$

we can write as $n \rightarrow \infty$

$$
\frac{h(n x)}{h(n)}=1+\frac{l_{1}(x, n)}{r(n)}+\frac{l_{2}(x, n)}{r(n)^{2}}+o\left(\frac{l_{2}(x, n)}{r(n)^{2}}\right)
$$

where in the range (6) the functions $l_{1}(x, n)$ and $l_{2}(x, n)$ are $o(r(n)) \epsilon$ for any $\epsilon>0$. c. As $x \rightarrow \infty, l_{1}^{\prime}(x, n)=o\left(x^{-\eta}\right)$ for some $\eta>0$. Under these conditions we can conclude [2] that the following asymptotic estimates are valid as $n \rightarrow \infty$ :

$$
\begin{aligned}
& \text { For } 0<\gamma<1 \text {, let } x \rightarrow 0, \quad x>\left[k_{\gamma} /(2-\epsilon) \lg r\left(b_{n}\right)\right]^{(1-\gamma) / \gamma} \text {. } \\
& \text { For } 1<\gamma<2 \text {, let } x \rightarrow-\infty,|x|<\left[(2-\epsilon) \lg r\left(b_{n}\right) / k_{\gamma}\right]^{(\gamma-1) / \gamma} ;
\end{aligned}
$$

then:

$$
\begin{array}{ll}
0<\gamma<1, & P\left(S_{n}<b_{n} x\right) \sim K_{\gamma} x^{\gamma / 2(1-\gamma)} \exp \left[-k_{\gamma} x^{-\gamma /(1-\gamma)}\right] \\
1<\gamma<2, & P\left(W_{n}<b_{n} x\right) \sim K_{\gamma}|x|^{-\gamma / 2(\gamma-1)} \exp \left[-k_{\gamma}|x|^{\gamma /(\gamma-1)}\right],
\end{array}
$$

where

$$
\begin{array}{ll}
0<\gamma<1, & k_{\gamma}=\Gamma(1-\gamma)^{1 /(1-\gamma)}\left\{\gamma^{\gamma /(1-\gamma)}-\gamma^{1 /(1-\gamma)}\right\}, \\
1<\gamma<2, & k_{\gamma}=\Gamma(-\gamma)^{-1 /(\gamma-1)}\left\{(1 / \gamma)^{1 /(\gamma-1)}-(1 / \gamma)^{\gamma /(\gamma-1)}\right\}
\end{array}
$$

and

$$
\begin{aligned}
& 0<\gamma<1, K_{\gamma}=\left[\Gamma(1-\gamma)^{1 /(1-\gamma)}\left(\gamma^{1 /(1-\gamma)}-\gamma^{(2-\gamma) /(1-\gamma)}\right) 2 \pi\right]^{-1 / 2}, \\
& 1<\gamma<2, K_{\gamma}=\left[\Gamma(-\gamma)^{-1 /(\gamma-1)} \gamma^{1(\gamma-1)}\left\{(1 / \gamma)^{\gamma /(\gamma-1)}-(1 / \gamma)^{(2 \gamma-1) /(\gamma-1)}\right\} 2 \pi\right]^{-1 / 2} .
\end{aligned}
$$

2. Introduction. In this paper we shall be interested in obtaining strong bounds for the sums $S_{n}$. Strong upper bounds were derived by Feller [3] from a more general theorem of his on strong bounds for random variables with infinite moments [4]. The same results had also been obtained by Marcinkiewicz [5] and Lévy [6] by more elementary considerations $\left({ }^{3}\right)$. Strong

(2) These conditions are satisfied, for instance, when $h(x)$ is of the following type: $(\lg x)^{p}$, $\exp (\lg \lg x)^{p}, \exp \exp \left(\lg _{3} x\right)^{p}, p>0$, etc.

(3) Lévy assumed in the case $1<\gamma<2$ that the first moment is zero. 
lower bounds were so far unknown except in a special case $[7 ; 8]$ with $\gamma=1 / 2$. We shall here obtain strong lower bounds for $S_{n}$. We are able to cover all cases in which the error term in the limiting approach to the stable distribution is of magnitude at most equal to $(\lg n)^{-2+\delta}$ for any $\delta>0$.

As was stated in $\$ 1$ the order of magnitude of the error is determined by the rate of growth of the function $h(x)$ in (1). It is possible that as the function $h(x)$ is allowed to increase more and more rapidly, the strong lower bounds which we obtain will need to be amended in a similar way as was done for the general law of the iterated logarithm [9]. We note, incidentally, that for $\gamma=2$, our reasoning gives this law for identically distributed positive r.v.

For the sake of completeness we state in $\$ 4$ the known theorems on strong upper bounds. They can also be obtained by the methods of this paper, the calculations being much simpler than those in $\$ 3$.

Random variables of the type dealt with appear, for instance, in the study of recurrent events. The r.v. $X_{k}$ here denotes the first recurrence time of this event and is always positive. If the recurrence time has infinite variance, Feller [3] shows that its distribution must be of such a type. The problem of determining strong lower bounds for sums of such r.v. was also raised by Feller. In $\$ 5$ we translate our results in terms of the r.v. $N_{n}$, the index of the $n$th recurrence of a recurrent event.

Our method of proof rests essentially on a lemma of Chung and Erdös [10]. This lemma states: Let $P(A \mid B)$ denote the conditional probability of $A$ given $B$; let $\bar{E}$ stand for the complement of $E$. Given then an infinite sequence of events $E_{1}, E_{2}, \cdots, E_{n}, \cdots$ satisfying:

(1) $\sum_{n=1}^{\infty} P\left(E_{n}\right)=\infty$.

(2) For every positive integer $h$ there exists a constant $c$, depending only on $h$, and an integral valued function $H(n)>n$, such that for every $k \geqq H(n)$ and $n \geqq h$, we have

$$
P\left(E_{k} \mid \bar{E}_{h} \cdots \bar{E}_{n}\right)>c P\left(E_{k}\right) .
$$

(3) There exist two constants $c_{1}$ and $c_{2}$ with the following property: to each $E_{j}$ there corresponds a set of events $E_{j_{1}}, \cdots, E_{j s}$ belonging to $\left\{E_{n}\right\}$ such that:

$$
\sum_{i=1}^{s} P\left(E_{j} E_{j_{i}}\right)<c_{1} P\left(E_{j}\right)
$$

and if $k>j$ but $E_{k}$ is not among the $E_{j_{i}}(1 \leqq i \leqq s)$ then

$$
P\left(E_{j} E_{k}\right)<c_{2} P\left(E_{j}\right) P\left(E_{k}\right) .
$$

Under these conditions it follows that

$$
P\left(E_{n} \text { i.o. }\right)=1 \text {, }
$$

where $E_{n}$ i.o. stands for "the event $E_{n}$ occurs for infinitely many $n$." 
Our proof in the case of convergence borrows part of its reasoning from Feller's proof of the general law of the iterated logarithm.

We should like to remark that although the results of this paper are simple, the necessary calculations are extremely laborious. The same is true for Feller's proof of the general law of the iterated logarithm. We do not know whether this complexity is inherent in this type of theorem; but we venture to hope that a deeper fundamental insight in to the mechanism of strong laws might simplify matters eventually.

\section{Strong lower bounds for $S_{n}$.}

Theorem I. Let $0<\gamma<1$, let $\psi(n) \uparrow \infty, \psi(n) / n \downarrow 0$; then

$$
P(\psi)=P\left(S_{n}<b_{n} / \psi\left(b_{n}\right)^{\left.1 / \gamma_{\text {i.o. }}\right)}=0 \text { or } 1\right.
$$

as

$$
I_{1}(\psi)=\int_{1}^{\infty}\left[\psi(x)^{1 / 2(1-\gamma)} / x\right] \exp \left[-k_{\gamma} \psi(x)^{1 /(1-\gamma)}\right] d x<\infty \text { or }=\infty .
$$

We henceforth write $\phi_{n}=\phi\left(b_{n}\right)$ for all functions under consideration. We first prove some lemmas.

Lемма a. If Theorem I holds for all functions $\psi(n)$ satisfying the following inequality for arbitrary positive $\epsilon$ :

$$
\psi(n) \leqq\left[(1+\epsilon) \lg \lg n / k_{\gamma}\right]^{1-\gamma}
$$

then it holds in general.

Proof. Case I: Let $g(n)$ be an arbitrary function satisfying the conditions of the theorem and $I_{1}(g)<\infty$. Denote the expression on the right-hand side of (10) by $\phi(n)$. Define $\Psi(x)=\min \{g(x), \phi(x)\}$. Then since clearly $I_{1}(\phi)<\infty$, we have $I_{1}(\Psi)<\infty$ and since $\Psi(n)$ satisfies (10)

$$
P\left(S_{n}<b_{n} / \Psi_{n}^{1 / \gamma} \text { i.o. }\right)=0
$$

and hence certainly

$$
P\left(S_{n}<b_{n} / g_{n}^{1 / \gamma} \text { i.o. }\right)=0 .
$$

Case II. Let $g(n)$ be an arbitrary function satisfying the conditions of the theorem and $I_{1}(g)=\infty$. Define again $\psi(x)=\min \{g(x), \phi(x)\}$. Now $I_{1}(\bar{\psi})=\infty$. Hence by the assumption of the lemma

$$
\begin{aligned}
& P\left(S_{n}<b_{n} / \psi_{n}^{1 / \gamma} \text { i.o. }\right)=1, \\
& P\left(S_{n}<b_{n} / \phi_{n}^{1 / \gamma} \text { i.o. }\right)=0 .
\end{aligned}
$$

Then there must exist an infinite sequence of indices $r_{1}, r_{2}, \ldots$ with $\Psi_{r_{i}}=g_{r_{i}}$ and 


$$
P\left(S_{r_{i}}<b_{r_{i}} / \Psi_{r_{i}} \text { i.o. }\right)=1 .
$$

Thus henceforth we consider only $\phi(x)$ satisfying (10).

Then by (8) and (9) of the introduction it follows that:

$$
P\left(S_{n}<b_{n} / \psi_{n}^{1 / \gamma}\right)=K_{\gamma} \psi_{n}^{-1 / 2(1-\gamma)}\left\{\exp \left[-k_{\gamma} \psi_{n}^{1 /(1-\gamma)}\right]\right\}(1+o(1)) .
$$

We define a sequence $\left\{n_{k}\right\}$ as follows: let $0<a<b$ be constants and

$$
b_{n_{k}}^{\gamma}\left(1+a / \psi_{n_{k}}^{1 /(1-\gamma)}\right) \leqq b_{n_{k+1}}^{\gamma}<b_{n_{k}}^{\gamma}\left(1+b / \psi_{n_{k}}^{1 /(1-\gamma)}\right) .
$$

Such a sequence exists since $\left(b_{n} / \psi_{n}\right) \rightarrow \infty$. We derive

LEMMA b. $I_{1}(\psi)$ converges or diverges with the sum

$$
\sum_{k} \psi_{n_{k}}^{-1 / 2(1-\gamma)} \exp \left[-k_{\gamma} \psi_{n_{k}}^{1 /(1-\gamma)}\right]=\sum_{k} P_{n_{k}}
$$

We shall repeatedly make use of these inequalities: let $0<x<1$, then $(x / 2)<\lg (1+x)<x$ and $2 x>-\lg (1-x)>x$. Write $b_{n}^{\gamma}=\beta_{n}, \beta_{n}-\beta_{n-1}=\sigma_{n}$, then

$$
\frac{2 \sigma_{n}}{\beta_{n}}>-\lg \left(\frac{\beta_{n}-\sigma_{n}}{\beta_{n}}\right)=\lg \left(\frac{\beta_{n}}{\beta_{n-1}}\right) \geqq \frac{\sigma_{n}}{\beta_{n}}
$$

and hence

$$
\sum_{n=n_{k}+1}^{n_{k+1}} \frac{\sigma_{n}}{\beta_{n}} \leqq \sum_{n=n_{k}+1}^{n_{k+1}} \lg \frac{\beta_{n}}{\beta_{n-1}}=\lg \frac{\beta_{n_{k+1}}}{\beta_{n_{k}}}<\lg \left(1+\frac{b}{\psi_{n_{k}}^{1 /(1-\gamma)}}\right)<\frac{b}{\psi_{n_{k}}^{1 /(1-\gamma)}}
$$

and

$$
\sum_{n=n_{k}+1}^{n_{k+1}} \frac{\sigma_{n}}{\beta_{n}} \geqq \frac{1}{2} \lg \frac{\beta_{n_{k+1}}}{\beta_{n_{k}}}>\frac{a}{4 \psi_{n_{k}}^{1 /(1-\gamma)}} .
$$

Now since $P_{n} \downarrow$ and $\psi_{n} \uparrow$ and $P_{n} \psi_{n}^{1 /(1-\gamma)} \downarrow 0$, we have for $n_{k}<n \leqq n_{k+1}$, $P_{n_{k+1}} \leqq P_{n}<P_{n_{k}}$ and

$$
\sum_{k} P_{n_{k}} \psi_{n_{k-1}}^{1 /(1-\gamma)} \sum_{n_{k-1}<n \leqq n_{k}} \frac{\sigma_{n}}{\beta_{n}} \leqq \sum_{n} \frac{\sigma_{n}}{\beta_{n}} P_{n} \psi_{n}^{1 /(1-\gamma)} \leqq \sum_{k} P_{n_{k}} \psi_{n_{k}}^{1 /(1-\gamma)} \sum_{n_{k-1}<n \leqq n_{k}} \frac{\sigma_{n}}{\beta_{n}} .
$$

These two sets of inequalities combine to give:

$$
\begin{aligned}
(a / 4) \sum_{k} \frac{1}{\psi_{n_{k}}^{1 / 2(1-\gamma)}} \exp \left[-k_{\gamma} \psi_{n_{k}}^{1 /(1-\gamma)}\right] & <\sum_{n} \frac{1}{\psi_{n}^{1 / 2(1-\gamma)}} \frac{\sigma_{n}}{\beta_{n}} \exp \left[-k_{\gamma} \psi_{n}^{1 /(1-\gamma)}\right] \\
& <b \sum_{k} \frac{1}{\psi_{n_{k}}^{1 / 2(1-\gamma)}} \exp \left[-k_{\gamma} \psi_{n_{k}}^{1 /(1-\gamma)}\right] .
\end{aligned}
$$


However the convergence or divergence of the integral $I_{1}(\psi)$ is determined by:

$$
\begin{aligned}
\int_{1}^{\infty}\left[\psi(x)^{1 / 2(1-\gamma)} / x\right] \exp & {\left[-k_{\gamma} \psi(x)^{1 /(1-\gamma)}\right] d x } \\
= & \sum_{n} \int_{\beta_{n-}}^{\beta_{n}} \frac{\psi(x)^{1 / 2(1-\gamma)}}{x} \exp \left[-k_{\gamma} \psi(x)^{1 /(1-\gamma)}\right] d x .
\end{aligned}
$$

The sum (15) is greater than

$$
\sum_{n}\left(\frac{\beta_{n}-\beta_{n-1}}{\beta_{n}}\right) \psi_{n}^{1 / 2(1-\gamma)} \exp \left[-k_{\gamma} \psi_{n}^{1 /(1-\gamma)}\right]
$$

and less than

$$
\sum_{n}\left(\frac{\beta_{n}-\beta_{n-1}}{\beta_{n-1}}\right) \psi_{n}^{1 / 2(1-\gamma)} \exp \left[-k_{\gamma} \psi_{n}^{1 /(1-\gamma)}\right]
$$

since taking into account the result of Lemma a, for $n$ sufficiently large the ratio $\left(\beta_{n-1} / \beta_{n}\right)$ will exceed $1 / 2$; it follows that these two sums converge and diverge together. Hence we compare them to the expression in the middle of (14) and obtain Lemma b.

Lemma c. For any constant $c$ we have:

$$
P\left(S_{n}<\left(b_{n} / \psi_{n}^{1 / \gamma}\right)\left(1+c / \psi_{n}^{1 /(1-\gamma)}\right)\right) \sim e^{k_{\gamma} \gamma c /(1-\gamma)} P\left(S_{n}<b_{n} / \psi_{n}^{1 / \gamma}\right)
$$

whereas for $c_{n} \rightarrow \pm \infty$, but $\left|c_{n} / \psi_{n}^{1 /(1-\gamma)}\right|<c<1-\eta$, we have:

(17) $P\left(S_{n}<\left(b_{n} / \psi_{n}^{1 / \gamma}\right)\left(1+c_{n} / \psi_{n}^{1 /(1-\gamma)}\right)\right) \sim C \exp \frac{k_{\gamma} \gamma c_{n}(1+\vartheta)}{1-\gamma} P\left(S_{n}<b_{n} / \psi_{n}^{1 / \gamma}\right)$ where $0<\vartheta<c(1-\gamma)^{-1}$.

We prove the second part of the lemma. The probability on the left of (17) equals by (8), (9), and (11):

$$
\begin{array}{r}
K_{\gamma}\left(1+c_{n} / \psi_{n}^{1 /(1-\gamma)}\right)^{\gamma / 2(1-\gamma)} \psi_{n}^{-1 / 2(1-\gamma)}\left\{\exp \left[-k_{\gamma} \psi_{n}^{1 /(1-\gamma)}\left(1+c_{n} / \psi_{n}^{1 /(1-\gamma)}\right)^{-\gamma /(1-\gamma)}\right]\right\} \\
\cdot(1+o(1)) .
\end{array}
$$

We expand the expression in the exponential into:

$$
-k_{\gamma} \psi_{n}^{1 /(1-\gamma)}\left[1-\frac{\gamma}{1-\gamma} \frac{c_{n}}{\psi_{n}^{1 /(1-\gamma)}}+\vartheta^{\prime} \frac{\gamma}{(1-\gamma)^{2}}\left(\frac{c_{n}}{\psi_{n}^{1 /(1-\gamma)}}\right)^{2-}\right]
$$

where $0<\vartheta^{\prime}<1$. Since $\left|c_{n}\right|<c \psi_{n}^{1 /(1-\gamma)}(17)$ follows. The result (16) is obtained more simply yet by a similar expansion. 
Let us then assume that the series (13) converges. With the aid of (16) we conclude that

$$
P\left(S_{n_{k}}<\left(b_{n_{k}} / \psi_{n_{k}}^{1 / \gamma}\right)\left(1+2 b / \gamma \psi_{n_{k}}^{1 /(1-\gamma)}\right) \text { i.o. }\right)=0 .
$$

Thus for almost all values assumed by the sequence of r.v. $X_{1}, X_{2}, \cdots, X_{n}$, and when $k$ is such that $n_{k} \leqq n<n_{k+1}$, the sums

$$
\begin{aligned}
S_{n} & \geqq S_{n_{k}}>\left(b_{n_{k}} / \psi_{n_{k}}^{1 / \gamma}\right)\left(1+2 b / \gamma \psi_{n_{k}}^{1 /(1-\gamma)}\right) \\
& >\left(b_{n_{k}} / b_{n_{k+1}}\right)\left(1-b / \gamma \psi_{n_{k}}^{1 /(1-\gamma)}\right)\left(1+2 b / \gamma \psi_{n_{k}}^{1 /(1-\gamma)}\right) \\
& >\left(b_{n} / \psi_{n}^{1 / \gamma}\right)\left(1-b / \gamma \psi_{n_{k}}^{1 /(1-\gamma)}\right)\left(1+2 b / \gamma \psi_{n_{k}}^{1 /(1-\gamma)}\right)>\left(b_{n} / \psi_{n}^{1 / \gamma}\right)
\end{aligned}
$$

and consequently

$$
P\left(S_{n}<b_{n} / \psi_{n}^{1 / \gamma} \text { i.o. }\right)=1 .
$$

We proceed to the case in which (13) diverges. In order to simplify our notation we write $k^{\prime}$ for $n_{k},(k+1)^{\prime}$ for $n_{k+1}$ etc. To prove that under the given circumstances $P\left(S_{n}<b_{n} / \psi_{n}^{1 / \gamma}\right.$ i.o. $)=1$, we define the events

$$
F_{k}: S_{k^{\prime}}<b_{k^{\prime}} / \psi_{k^{\prime}}^{1 / \gamma}
$$

and show that the conditions (1), (2), (3) of the lemma of Chung-Erdös are satisfied for these events.

Condition (1): $\sum_{k} P\left(F_{k}\right)=\infty$ follows from (11) since (13) diverges.

Condition (2): Consider the probability $P\left(F_{k} \mid \bar{F}_{h}, \cdots, \bar{F}_{m}\right)$ for any $h=1, \cdots, m$ and for $k \geqq H(m)$, where $H(m)$ is to be determined. Since $S_{k^{\prime}-m^{\prime}}^{\prime}=S_{k^{\prime}}-S_{m^{\prime}}$ is independent of $S_{m^{\prime}}$ it follows that:

$$
\begin{aligned}
P\left(F_{k} \mid \bar{F}_{h} \cdots \bar{F}_{m}\right) & \geqq P\left(F_{k}, S_{m^{\prime}}<b_{m^{\prime}}^{3} \mid \bar{F}_{h} \cdots \bar{F}_{m}\right) \\
& =P\left(S_{m^{\prime}}+S_{k^{\prime}-m^{\prime}}^{\prime}<\left(b_{k^{\prime}} / \psi_{k^{\prime}}^{1 / \gamma}\right)-b_{m^{\prime}}^{3}, S_{m^{\prime}}^{3}<b_{m^{\prime}}^{3} \mid \bar{F}_{h} \cdots \bar{F}_{m}\right) \\
& >P\left(S_{k^{\prime}-m^{\prime}}^{\prime}<\left(b_{k^{\prime}} / \psi_{k^{\prime}}^{1 / \gamma}\right)-b_{m^{\prime}}^{3}\right) P\left(S_{m^{\prime}}<b_{m^{\prime}}^{3} \mid \bar{F}_{h} \cdots \bar{F}_{m}\right) .
\end{aligned}
$$

The second term in the product equals:

$$
\begin{aligned}
1-P\left(S_{m^{\prime}}\right. & \left.\geqq b_{m^{\prime}}^{3}, \bar{F}_{h} \cdots \bar{F}_{m}\right) / P\left(\bar{F}_{h} \cdots \bar{F}_{m}\right) \\
& \geqq 1-P\left(S_{m^{\prime}} \geqq b_{m^{\prime}}^{3}\right) / P\left(\bar{F}_{h} \cdots \bar{F}_{m}\right) .
\end{aligned}
$$

We shall prove that $P\left(\bar{F}_{h} \cdots \bar{F}_{m}\right)>b_{m^{\prime}}^{-\gamma}$. From this follows then, since $P\left(S_{m^{\prime}} \geqq b_{m^{\prime}}^{3}\right)<b_{m^{\prime}}^{-2 \gamma}(1+o(1))$ by (4), that for $m$ sufficiently large (20) will be greater than $1-b_{m^{\prime}}^{-2 \gamma} \cdot b_{m^{\prime}}^{\gamma}>1-\epsilon$. Now the probability under study is given by:

$$
P\left(S_{h^{\prime}} \geqq b_{h^{\prime}} / \psi_{h^{\prime}}^{1 / \gamma}, \cdots, S_{m^{\prime}} \geqq b_{m^{\prime}} / \psi_{m^{\prime}}^{1 / \gamma}\right) \geqq \prod_{j=h}^{m} P\left(S_{j^{\prime}} \geqq b_{j^{\prime}} / \psi_{j^{\prime}}^{1 / \gamma}\right)
$$


since the r.v. are positive. But this product equals

$$
\prod_{j=h}^{m}\left(1-\left\{\exp \left[-k_{\gamma} \psi_{j^{\prime}}^{1 /(1-\gamma)}\right]\right\} / \psi_{j^{\prime}}^{1 / 2(1-\gamma)}\right)(1+o(1)) .
$$

However for large $j,\left\{\exp \left[-k_{\gamma} \psi_{j^{\prime}}^{1 /(1-\gamma)}\right]\right\}(1+o(1))<(a / 2) \psi_{j^{\prime}}^{-1 / 2(1-\gamma)}$ hence the above exceeds

$$
\prod_{j=h}^{m}\left(1-a / 2 \psi_{i^{\prime}}^{1 /(1-\gamma)}\right) .
$$

But by (12), $\left(1+a / \psi_{f^{1 /(1-\gamma)}}\right)^{-1} \geqq b_{j^{\prime}}^{\gamma} / b_{(+1)^{\prime}}^{\gamma}$. Expansion of the left-hand side of this inequality and comparison with the terms of the above product indicates that this product exceeds $b_{h}^{\gamma}, / b_{m^{\prime}}^{\gamma}>b_{m^{\prime}}^{-\gamma}$.

Consider now the expression

$$
\begin{aligned}
P\left(S_{k^{\prime}-m^{\prime}}^{\prime}\right. & \left.<\left(b_{k^{\prime}} / \psi_{k^{\prime}}^{1 / \gamma}\right)-b_{m^{\prime}}^{3}\right)>P\left(S_{k^{\prime}}<\left(b_{k^{\prime}} / \psi_{k^{\prime}}^{1 / \gamma}\right)-b_{m^{\prime}}^{3}\right) \\
& =P\left(S_{k^{\prime}}<\frac{b_{k^{\prime}}}{\psi_{k^{\prime}}^{1 / \gamma}}\left(1-\frac{b_{m^{\prime}}^{3}}{b_{k^{\prime}}} \psi_{k^{\prime}}^{1 / \gamma}\right)\right) .
\end{aligned}
$$

If now we choose $k$ large enough so that $\left(b_{m^{\prime}}^{3} \psi_{k^{\prime}}^{1 / \gamma} / b_{k^{\prime}}\right)<c / \psi_{k^{\prime}}^{1 /(1-\gamma)}$, then by Lemma c the probability (22) will exceed: $C_{1} P\left(S_{k^{\prime}}<b_{k^{\prime}} / \psi_{k^{\prime}}^{1 / \gamma}\right.$ and hence $P\left(F_{k} \mid \bar{F}_{h} \cdots \bar{F}_{m}\right)>(1-\epsilon) C_{1} P\left(F_{k}\right)$. The condition mentioned can be reduced to

$$
b_{k^{\prime}}>K b_{m^{\prime}}^{8} \psi_{k^{\prime}}^{1 / \gamma(1-\gamma)}
$$

Since $\psi_{k^{\prime}}<\left(\lg b_{k^{\prime}}\right)^{1-\gamma}$, this condition can be satisfied for all $k \geqq H(m)$, by choosing $H(m)$ so that $b_{k^{\prime}}>b_{m^{\prime}}^{4}$. This will certainly be true if $H(m)>m^{\prime}+\lg m^{\prime}$ as can be seen from the definition (12) of the sequence $\left\{n_{k}\right\}$.

LEMMA d. Let $I_{1}(\psi)=\infty$. If Theorem I holds for all $\psi(n)$ satisfying

$$
\psi(n) \geqq\left(\lg \lg n / 2 k_{\gamma}\right)^{1-\gamma},
$$

then the theorem holds in general.

Proof. Let $g(n)$ be an arbitrary function satisfying the conditions of the theorem and $I_{1}(g)=\infty$. Call the expression on the right of the inequality (24), $\phi(n)$. Let $\Psi(x)=\max (g(x), \phi(x))$. Then $\Psi(n)$ satisfies (24). If we can show that $I_{1}(\Psi)=\infty$ then it will follow from the hypothesis that $P\left(S_{n}<b_{n} / \psi_{n}^{1 / \gamma}\right.$ i.o. $)=1$, and certainly whenever $S_{n}<b_{n} / \psi_{n}^{1 / \gamma}$ we have also that $S_{n}<b_{n} / g_{n}^{1 / \gamma}$; hence the lemma. Let us then assume that $I_{1}(\bar{\psi})<\infty$ which will lead to a contradiction. Now $I_{1}(\Psi)<\infty$ implies the existence of an infinite sequence of indices $n_{1}, n_{2}, \cdots$ with $\Psi\left(n_{i}\right)=g\left(n_{i}\right)>\left(\lg \lg n_{i} / k_{\gamma}\right)^{1-\gamma}$. On the other hand since $I_{1}(g)=\infty$, there must exist an infinite sequence of indices $m_{1}, m_{2}, \cdots$ with 
$g\left(m_{i}\right) \neq \bar{\psi}\left(m_{i}\right)$ and thus with $g\left(m_{i}\right) \leqq\left(\lg \lg m_{i} / 2 k_{\gamma}\right)^{1-\gamma}=\phi\left(m_{i}\right)$. Let then $n_{i_{k}}$ and $m_{\boldsymbol{i}^{\prime}}$ be indices such that:

$$
\begin{aligned}
g\left(n_{i_{k}}\right) & >\left(\lg \lg n_{i_{k}} / k_{\gamma}\right)^{1-\gamma}, \\
\left(\lg \lg n / k_{\gamma}\right)^{1-\gamma} & \geqq g(n)>\left(\lg \lg n / 2 k_{\gamma}\right)^{1-\gamma} \text { for } n_{i_{k}}<n<m_{i_{k^{\prime}}}
\end{aligned}
$$

and

$$
g\left(m_{i_{k^{\prime}}}\right) \leqq\left(\lg \lg m_{i_{k^{\prime}}} / 2 k_{\gamma}\right)^{1-\gamma} .
$$

There exists an infrnite number of such $k$ by the discussion above. We recall that $g(n)$ is a monotonically increasing function, since it is to satisfy the conditions of Theorem $I$, and hence

$$
\left(\lg \lg n_{i_{k}} / k_{\gamma}\right)^{1-\gamma}<\left(\lg \lg m_{i_{k^{\prime}}} / 2 k_{\gamma}\right)^{1-\gamma}
$$

or

$$
m_{i_{k^{\prime}}}>\left(n_{i_{k}}\right)^{\lg n_{i_{k} .}}
$$

However for all $n$ in the range $n_{i_{k}}<n<m_{i_{k}}$ we have $\psi(n)=g(n)$ and thus by the above:

$$
\begin{aligned}
I_{1}(\Psi) & >\sum_{k} \sum_{n=n_{i_{k^{\prime}}}}^{m_{i_{k^{\prime}}}} \frac{\Psi_{n}^{1 / 2(1-\gamma)}}{n+1} \exp \left[-k_{\gamma} \Psi(n+1)^{1 /(1-\gamma)}\right] \\
& >\sum_{k} \sum_{n=n_{i_{k}}}^{m_{k_{k \prime}}} \frac{(\lg \lg n)^{1 / 2(1-\gamma)}}{2^{1 / \gamma} k^{1 / \gamma}(n+1) \lg (n+1)} \\
& >C \sum_{k}\left(\lg \lg \left(n_{i_{k}}\right) \lg ^{\lg n_{i_{k}}}-\lg \lg n_{i_{k}}\right) \\
& >C \sum_{k} \lg \lg n_{i_{k}}=\infty
\end{aligned}
$$

Lemma e. For $m>r$, and $k, j, l$ and $t$ positive quantities:

$$
\begin{gathered}
P\left(S_{r}<k, S_{m}<j\right)<P\left(S_{r}<k\right) P\left(S_{m-r}<j\right), \\
P\left(t<S_{r}<k, S_{m}<l\right)<P\left(S_{r}<k P\left(S_{m-r}<l-t\right) .\right.
\end{gathered}
$$

Proof. If we let $F_{n}(x)$ denote the distribution function of $S_{n}$, the left-hand sides of (29) and (30) equal respectively

$$
\begin{aligned}
& \int_{0}^{k} F_{m-r}(j-x) d F_{r}(x)<P\left(S_{r}<k\right) P\left(S_{m-r}<j\right), \\
& \int_{t}^{k} F_{m-r}(l-x) d F_{r}(x)<P\left(S_{r}<k\right) P\left(S_{m-r}<l-t\right)
\end{aligned}
$$

when we recall that the random variables are positive. Let now 


$$
P_{r}=P\left(S_{n_{k}}<b_{n_{k}} / \psi_{n_{k}}^{1 / \gamma}, S_{n_{r}}<b_{n_{r}} / \psi_{n_{r}}^{1 / \gamma}\right) .
$$

By (29) this probability is less than

$$
P\left(S_{n_{k}}<b_{n_{k}} / \psi_{n_{k}}^{1 / \gamma}\right) P\left(S_{n_{r}-n_{k}}<b_{n_{r}} / \psi_{n_{r}}^{1 / \gamma}\right) .
$$

Now consider all $r$ for which:

$$
n_{r}>c_{1} n_{k} \psi_{n_{r}}^{1 /(1-\gamma)}
$$

then for such $r$,

$$
\left(n_{r}-n_{k}\right)>n_{r}\left(1-c_{2} / \psi_{n_{r}}^{1 /(1-\gamma)}\right)
$$

and

$$
\frac{h\left(n_{r}-n_{k}\right)}{h\left(n_{r}\right)}=\frac{h\left[n_{r}\left(1-n_{k} / n_{r}\right)\right]}{h\left(n_{r}\right)}=1+O\left(\frac{1}{r\left(n_{r}\right)^{1-\epsilon}}\right)
$$

by (7); for by (32), $l>1-\left(n_{k} / n_{r}\right)>1-c_{1} / \psi_{n_{r}}^{1 /(1-\gamma)}$. Then

$$
\begin{aligned}
b_{n_{r}-n_{k}} & =\left(n_{r}-n_{k}\right)^{1 / \gamma}\left(h\left(n_{r}-n_{k}\right)\right)^{1 / \gamma}>n_{r}^{1 / \gamma}\left(h\left(n_{r}\right)^{1 / \gamma}\left(1-c_{3} / \psi_{n_{r}}^{1 /(1-\gamma)}\right)\right) \\
& >b_{n_{r}}\left(1-c_{3} / \psi_{n_{r}}^{1 /(1-\gamma)}\right) .
\end{aligned}
$$

Hence the second probability in the product (31) will be less than:

$$
\begin{aligned}
P\left(S_{n_{r}-n_{k}}\right. & \left.<\left(b_{n_{r}-n_{k}} / \psi_{n_{r}}^{1 / \gamma}\right)\left(1-c_{3} / \psi_{n_{r}}^{1 /(1-\gamma)}\right)\right) \\
& <C_{1} e^{\gamma c k_{\gamma} /(1-\gamma)} P\left(S_{n_{r}-n_{k}}<b_{n_{r}-n_{k}} / \psi_{n_{r}}^{1 / \gamma}\right)
\end{aligned}
$$

by (16). Thus by (8), (9), and (33) it follows that

$$
\text { (31) }<C_{2} P\left(S_{n_{k}}<b_{n_{k}} / \psi_{n_{k}}^{1 / \gamma}\right) P\left(S_{n_{r}}<b_{n_{r}} / \psi_{n_{r}}^{1 / \gamma}\right) .
$$

We next proceed to the case in which (32) does not hold, i.e.

$$
n_{r}<c_{1} n_{k} \psi_{n_{r}}^{1 /(1-\gamma)}
$$

We call the set of indices in which (35) is valid the set $Q_{k}$. Applying (7) we have for $r \in Q_{k}$ :

$$
\begin{aligned}
\frac{h\left(n_{r}\right)}{h\left(n_{k}\right)} & =\frac{h\left(n_{r}\right)}{h\left(n_{r}\left(n_{k} / n_{r}\right)\right)}=1+O\left(\frac{1}{r\left(n_{r}\right)^{1-\epsilon}}\right)=1+O\left(r\left(n_{k}\right)^{-1+\epsilon}\right), \\
\frac{h\left(n_{r}\right)}{h\left(n_{r}-n_{k}\right)} & =\frac{h\left(n_{r}\right)}{h\left(n_{r}\left(1-\left(n_{k} / n_{r}\right)\right)\right.}=1+O\left(\frac{1}{r\left(n_{r}\right)^{1-\epsilon}}\right), \\
\frac{h\left(n_{r}-n_{k} !\right.}{h\left(n_{k}\right)} & =\frac{h\left(n_{k}\left(n_{r} / n_{k}\right)-1\right)}{h\left(n_{k}\right)}=1+O\left(\frac{1}{r\left(n_{k}\right)^{1-\epsilon}}\right) .
\end{aligned}
$$


The required relations (6) are satisfied in these three cases by virtue of the following inequalities derived from (10), (12), (24), and (35): $1>\left(n_{k} / n_{r}\right)$ $>1 / c_{1} \psi_{n_{r}}^{1 /(1-\gamma)} ; 1>1-\left(n_{k} / n_{r}\right)>1-\left(n_{k} / n_{k+1}\right)>1-c_{4} / \psi_{n_{k}}^{1 /(1-\gamma)} ; c_{5} \psi_{n_{k}}^{1 /(1-\gamma)}>c_{1} \psi_{n_{r}}^{1 /(1-\gamma)}$ $>\left(n_{r} / n_{k}\right)-1>\left(n_{k+1} / n_{k}\right)-1>c_{6} / \psi_{n_{k}}^{1 /(1-\gamma)}$.

From (12) we now derive the double inequality:

$$
b_{n_{k}}^{\gamma} \prod_{j=k}^{r}\left(1+a / \psi_{n_{j}}^{1 /(1-\gamma)}\right) \leqq b_{n_{r}}^{\gamma}<b_{n_{k}}^{\gamma} \prod_{j=k}^{r}\left(1+b / \psi_{n_{j}}^{1 /(1-\gamma)}\right) .
$$

For $r \in Q_{k}$ the left-hand inequality leads to:

$$
\begin{aligned}
n_{k} & \leqq n_{r}\left[h\left(n_{r}\right) / h\left(n_{k}\right)\right] /\left(1+a / \psi_{n_{r}}^{1 /(1-\gamma)}\right)^{r-k} \\
& \leqq n_{r}\left(1-a(r-k) / 4 \psi_{n_{r}}^{1 /(1-\gamma)}\right)\left(1+O\left(r\left(n_{r}\right)^{1-\epsilon}\right)\right)
\end{aligned}
$$

and hence:

$$
(r-k)<(4 / a)\left(\left(n_{r}-n_{k}\right) / n_{r}\right) \psi_{n_{r}}^{1 /(1-\gamma)}<(4 / a) \psi_{n_{r}}^{1 /(1-\gamma)} .
$$

With the aid of (24) we then obtain an upper bound to the number of indices contained in $Q_{k}$; i.e. for $r \in Q_{k}$ :

$$
(r-k)<C_{3}\left(\lg \lg b_{n_{k}}\right)^{1+\delta}, \quad 1>\delta>0 .
$$

We consider first the subset $Q_{k}^{1}$ of $Q_{k}$ for which:

$$
b_{n_{r}}<b_{n_{r}-n_{k}} \mu^{1 / \gamma}
$$

where $\mu$ is a constant exceeding $64(1+\epsilon) b / a$. The probability (31) will now te less than

$$
P\left(S_{n_{k}}<b_{n_{k}} / \psi_{n_{k}}^{1 / \gamma}\right) P\left(S_{n_{r}-n_{k}}<b_{n_{r}-n_{k}} \mu^{1 / \gamma} / \psi_{n_{r}}^{1 / \gamma}\right) .
$$

Hence we conclude with the aid of (10) and (24):

$$
\begin{aligned}
\sum_{r \in Q_{k}{ }^{1}} P_{r} & <P\left(S_{n_{k}}<b_{n_{k}} / \psi_{n_{k}}^{1 / \gamma}\right) \sum_{r \in Q_{k}} \exp \left[-k_{\gamma} \psi_{n_{r}}^{1 /(1-\gamma)} \mu^{-1 /(1-\gamma)}\right] \\
& <P\left(S_{n_{k}}<b_{n_{k}} / \psi_{n_{k}}^{1 / \gamma}\right) \sum_{r \in Q_{k}} \exp \left[-p_{\gamma} \lg \lg b_{n_{k}}\right] \\
& <P\left(S_{n_{k}}<b_{n_{k}} / \psi_{n_{k}}^{1 / \gamma}\right) \sum_{r \in Q_{k}}\left(\lg b_{n_{k}}\right)^{-p_{\gamma}} \\
& <C_{4} P\left(S_{n_{k}}<b_{n_{k}} / \psi_{n_{k}}^{1 / \gamma}\right)\left(\lg \lg b_{n_{k}}\right)^{1+\delta}\left(\lg b_{n_{k}}\right)^{-p_{\gamma}} \\
& <M_{1} P\left(S_{n_{k}}<b_{n_{k}} / \psi_{n_{k}}^{1 / \gamma}\right)
\end{aligned}
$$

for $k$ large enough, $p_{\gamma}=\mu^{-1 /(1-\gamma)} / 2$. We next consider the set $Q_{k}^{2} \in Q_{k}$ for which

$$
b_{n_{r}}>b_{n_{r}-n_{k}} \mu^{1 / \gamma}
$$

or 


$$
\begin{aligned}
n_{r}-n_{k} & <n_{r} h\left(n_{r}\right) / \mu h\left(n_{r}-n_{k}\right) \\
& <2 n_{r} / \mu
\end{aligned}
$$

by (37). For $r \in Q_{k}^{2}$, (40) and (45) lead to:

$$
\begin{aligned}
(r-k) & <8 \psi_{n_{r}}^{1 /(1-\gamma)} / a \mu \\
& <(1+\epsilon) 16 \lg \lg n_{k} / k_{\gamma} a \mu \\
& <\psi_{n_{k}}^{1 /(1-\gamma) / 2 b}
\end{aligned}
$$

since $\mu$ was chosen greater than $64(1+\epsilon) b / a$. We are now allowed to expand the right-hand side of the product (39) to obtain

$$
b_{n_{k}}^{\gamma}\left(1+a(r-k) / \psi_{n_{r}}^{1 /(1-\gamma)}\right) \leqq b_{n_{r}}^{\gamma}<b_{n_{k}}^{\gamma}\left(1+2 b(r-k) / \psi_{n_{k}}^{1 /(1-\gamma)}\right) .
$$

Let $A$ be a constant $<b$. Consider

$$
\begin{aligned}
P_{r}^{\prime} & =P\left(S_{n_{k}}<\left(b_{n_{k}} / \psi_{n_{k}}^{1 / \gamma}\right)\left(1-(r-k) A / \psi_{n_{k}}^{1 /(1-\gamma)}\right), S_{n_{r}}<b_{n_{r}} / \psi_{n_{r}}^{1 / \gamma}\right) \\
& <P\left(S_{n_{k}}<\left(b_{n_{k}} / \psi_{n_{k}}^{1 / \gamma}\right)\left(1-(r-k) A / \psi_{n_{k}}^{1 /(1-\gamma)}\right) .\right.
\end{aligned}
$$

We apply Lemma c, (17), to this probability, noting that by (46):

and obtain:

$$
A(r-k) / \psi_{n_{k}}^{1 /(1-\gamma)}<1 / 2,
$$

$$
P_{r}^{\prime}<C_{5}\left\{\exp \left[-k_{\gamma}(r-k) A \gamma(1+\vartheta) /(1-\gamma)\right]\right\} P\left(S_{n_{k}}<b_{n_{k}} / \psi_{n_{k}}^{1 / \gamma}\right)
$$

and

$$
\begin{aligned}
\sum_{r \in Q_{k}^{2}} P_{r}^{\prime} & <C_{6} P\left(S_{n_{k}}<b_{n_{k}} / \psi_{n_{k}}^{1 / \gamma}\right) \sum_{r=k+1}^{\infty} \exp \left[-c_{7}(r-k)\right] \\
& <M_{2} P\left(S_{n_{k}}<b_{n_{k}} / \psi_{n_{k}}^{1 / \gamma}\right) .
\end{aligned}
$$

Finally we consider:

$$
\sum_{r \in Q_{k}^{2}} P\left(\left(b_{n_{k}} / \psi_{n_{k}}^{1 / \gamma}\right)\left(1-(r-k) A / \psi_{n_{k}}^{1 /(1-\gamma)}\right)<S_{n_{k}}<b_{n_{k}} / \psi_{n_{k}}^{1 / \gamma}, S_{n_{r}}<b_{n_{r}} / \psi_{n_{r}}^{1 / \gamma}\right) .
$$

By (30) this is less than

$$
\begin{aligned}
P\left(S_{n_{k}}<\right. & \left.b_{n_{k}} / \psi_{n_{k}}^{1 / \gamma}\right) \sum_{r \in Q_{k}^{2}} P\left(S_{n_{r}-n_{k}}<\left(b_{n_{r}} / \psi_{n_{r}}^{1 / \gamma}\right)-\left(b_{n_{k}} / \psi_{n_{k}}^{1 / \gamma}\right)\right. \\
& \left.\cdot\left(1-(r-k) A / \psi_{n_{k}}^{1 /(1-\gamma)}\right)\right) .
\end{aligned}
$$

From (47) follows that: 


$$
\begin{array}{ll}
\left(b_{n_{r}} / \psi_{n_{r}}^{1 / \gamma}\right)-\left(b_{n_{k}} / \psi_{n_{k}}^{1 / \gamma}\right)\left(1-(r-k) A / \psi_{n_{k}}^{1 /(1-\gamma)}\right) \\
\quad<\left(b_{n_{k}} / \psi_{n_{k}}^{1 / \gamma}\right)\left(\left(1+2 b(r-k) / \psi_{n_{k}}^{1 /(1-\gamma)}\right)-1-(r-k) A / \psi_{n_{k}}^{1 /(1-\gamma)}\right) \\
(50) \quad<\left(b_{n_{k}} / \psi_{n_{k}}^{1 / \gamma(1-\gamma)}\right)(r-k)\left(\left(2^{(1 / \gamma)+1} b / \gamma\right)+A\right) .
\end{array}
$$

From (40) follows

$$
n_{r}-n_{k}>(a / 4)(r-k) n_{r} / \psi_{n_{r}}^{1 /(1-\gamma)}>(a / 4)(r-k) n_{k} / \psi_{n_{r}}^{1 /(1-\gamma)} .
$$

Hence

$$
\begin{aligned}
b_{n_{r}-n_{k}}> & {\left[\left(\frac{a}{4}\right)\left(\frac{r-k}{\psi_{n_{r}}^{1 /(1-\gamma)}}\right)\left(\frac{h\left(n_{r}-n_{k}\right)}{h\left(n_{k}\right)}\right)\right]^{1 / \gamma} b_{n_{k}} } \\
> & (a / 8)^{1 / \gamma}\left((r-k)^{1 / \gamma} / \psi_{n_{r}}^{1 / \gamma(1-\gamma)}\right) b_{n_{k}}
\end{aligned}
$$

by (38). The probability in (49) is then less than:

$$
\begin{aligned}
P\left(S_{n_{k}}\right. & \left.<b_{n_{k}} / \psi_{n_{k}}^{1 / \gamma}\right) \sum_{r \in Q_{k^{2}}} P\left(S_{n_{r}-n_{k}}<b_{n_{r}-n_{k}} c_{8} /(r-k)^{(1-\gamma) / \gamma}\right) \\
& <P\left(S_{n_{k}}<b_{n_{k}} / \psi_{n_{k}}^{1 / \gamma}\right) \sum_{r=k}^{\infty} \exp -c_{8} k_{\gamma}(r-k) \\
& <M_{3} P\left(S_{n_{k}}<b_{n_{k}} / \psi_{n_{k}}^{1 / \gamma}\right) .
\end{aligned}
$$

The inequalities (43), (48), and (52) together prove that:

$$
\sum_{r \in Q_{k}} P_{r}<\left(M_{1}+M_{2}+M_{8}\right) P\left(S_{n_{k}}<b_{n_{k}} / \psi_{n_{k}}^{1 / \gamma}\right),
$$

and together with (34) they verify condition $\mathrm{c}$ of the lemma of Chung-Erdös.

Theorem II. Let $1<\gamma<2$, let $\psi(n) \uparrow \infty, \psi(n) / n \downarrow 0$; then

$$
P(\psi)=P\left(S_{n}-n \mu<-b_{n} \psi\left(b_{n}\right)^{\left.1 / \gamma_{\text {i.o. }}\right)}=0 \text { or } 1\right.
$$

as

$$
I_{2}(\psi)=\int_{1}^{\infty} \frac{\psi(x)^{1 / 2(\gamma-1)}}{x} \exp \left[-k_{\gamma} \psi(x)^{1 /(\gamma-1)}\right] d x<\infty \text { or }=\infty .
$$

The proof of this theorem does not differ appreciably from that of Theorem I. We shall only work out the points of difference between the two proofs. We again write $\psi_{n}$ for $\psi\left(b_{n}\right)$.

LEMMA a' AND LEMMA $\mathrm{d}^{\prime}$. If Theorem II holds for all functions $\psi(n)$ satisfying the following inequalities: 


$$
\left(\lg \lg n / 2 k_{\gamma}\right)^{\gamma-1} \leqq \psi(n)<\left((1+\epsilon) \lg \lg n / k_{\gamma}\right)^{\gamma-1}
$$

then it holds in general.

We have by (6) and (8) when we assume henceforth that $\psi(n)$ satisfies (53):

$$
P\left(W_{n}<-b_{n} \psi_{n}\right)=K_{\gamma} \psi_{n}^{-1 / 2(\gamma-1)}\left\{\exp \left[-k_{\gamma} \psi_{n}^{1 /(\gamma-1)}\right]\right\}(1+o(1)) .
$$

We define a sequence $n_{k}$ by

$$
b_{n_{k}}^{\gamma}\left(1+a / \psi_{n_{k}}^{1 /(\gamma-1)}\right) \leqq b_{n_{k+1}}^{\gamma}<b_{n_{k}}^{\gamma}\left(1+b / \psi_{n_{k}}^{1 /(\gamma-1)}\right) .
$$

LEMMA $\mathrm{b}^{\prime} . I_{2}(\psi)$ converges or diverges with:

$$
\sum_{k} \psi_{n_{k}}^{1 / 2(\gamma-1)} \exp \left[-k_{\gamma} \psi_{n_{k}}^{1 /(\gamma-1)}\right] .
$$

LemMa $\mathrm{c}^{\prime}$. For any constant $c$ we have:

$$
P\left(W_{n}<-b_{n} \psi_{n}^{1 / \gamma}\left(1-c / \psi_{n}^{1 /(\gamma-1)}\right)\right) \sim C e^{\gamma c k_{\gamma} /(\gamma-1)} P\left(W_{n}<-b_{n} \psi_{n}^{1 / \gamma}\right)
$$

whereas for $c_{n} \rightarrow \pm \infty$, but $\left|c_{n} / \psi_{n}^{1 /(\gamma-1)}\right|<c<1-\eta$, we have

(58) $P\left(W_{n}<-b_{n} \psi_{n}^{1 / \gamma}\left(1-c_{n} / \psi_{n}^{1 /(\gamma-1)}\right)\right) \sim C \exp \frac{\gamma c k_{\gamma} c_{n}(1+\vartheta)}{\gamma-1} P\left(W_{n}<-b_{n} \psi_{n}^{1 / \gamma}\right)$ where $0<\vartheta<c(\gamma-1)^{-1}$.

The proof of these four lemmas follows the exact lines of the corresponding lemmas for the case $0<\gamma<1$. To proceed to the proof of the theorem, we first consider the case of convergence, i.e. we assume that the series (56) converges. With the aid of Lemma $c^{\prime}$ we then conclude that

$$
P\left(W_{n_{k}}<-\left(b_{n_{k}} \psi_{n_{k}}^{1 / \gamma}\right)\left(1+b / \psi_{n_{k}}^{1 /(\gamma-1)}\right) \text { i.o. }\right)=0 .
$$

We define the following events for $n_{k} \leqq n<n_{k+1}$ :

$$
\begin{aligned}
& A_{n}: W_{n}<-b_{n} \psi_{n}^{1 / \gamma}, \\
& B_{n k}: W_{n_{k+1}}-W_{n}<b_{n_{k+1}-n} 2^{1 / \gamma} b^{(\gamma-1) / \gamma}, \\
& C_{k}: W_{n_{k+1}}<-b_{n_{k+1}} \psi_{n_{k}}^{1 / \gamma}\left(1+b / \gamma \psi_{n_{k}}^{1 /(\gamma-1)}\right) .
\end{aligned}
$$

If we can show that (59) implies that for $N$ sufficiently large:

$$
P\left(\sum_{n=N}^{\infty} A_{n}\right)<\epsilon
$$

it would follow that 


$$
P\left(W_{n}<-b_{n} \psi_{n}^{1 / \gamma} \text { i.o. }\right)=0,
$$

and thus the first part of Theorem II would be proven. From (55) we deduce, since $b_{n}=n^{1 / \gamma} h(n)^{1 / \gamma}$,

$$
\frac{n_{k+1}}{n_{k}}<\left(1+\frac{2^{\gamma-1} b}{\psi_{n_{k}}^{1 /(\gamma-) 1}}\right) .
$$

Thus for $n_{k} \leqq n<n_{k+1}$,

$$
b_{n_{k+1}-n} \leqq b_{n_{k+1}-n_{k}}<b_{n_{k}} 2^{1-1 / \gamma} b^{1 / \gamma} / \psi_{n_{k}}^{1 / \gamma(\gamma-1)} .
$$

Also, since by (1) the distribution of $W_{n_{k+1}-n}$ approximates the stable distribution:

$$
\begin{aligned}
P\left(B_{n k}\right) & =P\left(W_{n_{k+1}-n}<-b_{n_{k+1}-n} 2^{1 / \gamma} b^{(\gamma-1) / \gamma}\right) \\
& \sim 1-\left(2 b^{\gamma-1}\right)^{-1}>1 / 2
\end{aligned}
$$

if $b>1$. At all points of the intersection of $A_{n} B_{n k}$ we have then:

$$
\begin{aligned}
W_{n_{k+1}}=W_{n}+W_{n_{k+1}-n} & <-b_{n} \psi_{n}^{1 / \gamma}+b_{n_{k}} 2 b / \psi_{n_{k}}^{1 / \gamma(\gamma-1)} \\
& <-b_{n_{k}} \psi_{n_{k}}^{1 / \gamma}\left(1-2 b / \psi_{n_{k}}^{1 /(\gamma-1)}\right) \\
& <-b_{n_{k+1}} \psi_{n_{k}}^{1 / \gamma}\left(1-b / 2 \psi_{n_{k}}^{1 /(\gamma-1)}\right)\left(1-2 b / \psi_{n_{k}}^{1 /(\gamma-1)}\right) \\
& <-b_{n_{k+1}} \psi_{n_{k}}^{1 / \gamma}\left(1+b / \psi_{n_{k}}^{1 /(\gamma-1)}\right)
\end{aligned}
$$

so that $C_{k} \supset A_{n} B_{n k}$ for all $n$ such that $n_{k} \leqq n<n_{k+1}$. On the other hand $A_{m}$ and $B_{n k}$ are independent whenever $m \leqq n$. Thus:

$$
\begin{aligned}
P\left(C_{k}\right) & \geqq P\left(\sum_{n=n_{k}+1}^{n_{k+1}} A_{n} B_{n k}\right)=\sum_{n=n_{k}+1}^{n_{k+1}} P\left(A_{n} B_{n k}-A_{n} B_{n k} \sum_{m=n_{k}+1}^{n-1} A_{m} B_{m k}\right) \\
& \geqq \sum_{n=n_{k}+1}^{n_{k}+1} P\left(A_{n} B_{n k}-A_{n} B_{n k} \sum_{m=n_{k}+1}^{n-1} A_{m}\right) \\
& =\sum_{n=n_{k}+1}^{n_{k+1}} P\left(B_{n k}\right) P\left(A_{n}-A_{n} \sum_{m=n_{k}+1}^{n-1} A_{m}\right) \\
& >\frac{1}{2} P\left(\sum_{n=n_{k}+1}^{n_{k+1}} A_{n}\right) .
\end{aligned}
$$

Thus

$$
P\left(\sum_{n=n_{k}+1}^{n_{k+1}} A_{n}\right)<2 P\left(C_{k}\right)
$$

But we know from (53) and (59) that $\sum_{k} P\left(C_{k}\right)<\infty$. The relation (60) then 
follows readily from the above inequality. Let now the series (59) diverge. We proceed to prove the second part of Theorem II. The points of difference with the case $0<\gamma<1$ in the proof of the validity of the conditions of ChungErdös arise from the fact that the random variables $W_{n}$ are now not necessarily positive. If we define $F_{k}$ as the event:

$$
F_{k}: W_{n_{k}}<-b_{n_{k}} \psi_{n_{k}}^{1 / \gamma}
$$

then condition (2) is verified exactly as before when we write $W_{n}$ for $S_{n}$ and $b_{n} \psi_{n}^{1 / \gamma}$ for $b_{n} / \psi_{n}^{1 / \gamma}$ throughout the proof. We change the condition $S_{m^{\prime}}<b_{m^{\prime}}^{3}$ into $W_{m^{\prime}}<b_{m^{\prime}}^{3}$ and obtain as before:

$$
P\left(F_{k} \mid \bar{F}_{h} \cdots \bar{F}_{m}\right)>P\left(W_{k^{\prime}-m^{\prime}}^{\prime}<-b_{k^{\prime}} \psi_{k^{\prime}}^{1 / \gamma}-b_{m^{\prime}}^{8}\right) P\left(W_{m^{\prime}}<b_{m^{\prime}}^{3} \mid \bar{F}_{h} \cdots \bar{F}_{m}\right) .
$$

The second term in the product exceeds $1-b_{m^{\prime}}^{-\gamma}$, by an argument like the one for the case $0<\gamma<1$. Choosing then $H(m)$ large enough so that

$$
\left(b_{k^{\prime}} / b_{k^{\prime}-m^{\prime}}\right)\left(1+b_{m^{\prime}}^{8} / \psi_{k^{\prime}}^{1 / \gamma} b_{k^{\prime}}\right)<1+c / \psi_{k^{\prime}}^{1 /(\gamma-1)}
$$

it will follow that

$$
P\left(F_{k} \mid \bar{F}_{h} \cdots \bar{F}_{m}\right)>(1-2 \epsilon) e^{\gamma c k}{ }_{\gamma}^{(\gamma-1)} C P\left(F_{k}\right) .
$$

Verification of conditions (3) of Chung-Erdös calls for a slightly different treatment than before.

LEMma f. Let $t, k$, and $l$ be greater than zero, then

$$
P\left(-t<W_{m}<-k, W_{r}<-l\right)<P\left(W_{m}<-k\right) P\left(\bar{W}_{r-m}<-l+t\right) \text {. }
$$

For if $F_{m}(x)$ is the distribution function of $W_{n}$, due to the independence of the random variables we can write the left-hand side as

$$
\int_{-t}^{-k} F_{r-m}(-l-x) d F_{m}(x) \leqq F_{m}(-k) F_{r-m}(-l+t)
$$

since the random variables $x$ are positive. Now again let

$$
P_{r}=P\left(W_{n_{k}}<-b_{n_{k}} \psi_{n_{k}}^{1 / \gamma}, W_{n_{r}}<-b_{n_{r}} \psi_{n_{r}}^{1 / \gamma}\right) .
$$

We note that for all $k, W_{n_{k}} \geqq-n_{k} \mu$. Thus by Lemma $\mathrm{f}$ :

$$
P_{r}<P\left(W_{n_{k}}<-b_{n_{k}} \psi_{n_{k}}^{1 / \gamma}\right) P\left(W_{n_{r}-n_{k}}<-b_{n_{r}} \psi_{n_{r}}^{1 / \gamma}+n_{k} \mu\right) .
$$

For

$$
b_{n_{r}}>b_{n_{k}} \psi_{n_{r}}^{1 / \gamma(\gamma-1)}>n_{k} \psi_{n_{r}}^{1 / \gamma(\gamma-1)}
$$

we have 


$$
-b_{n_{r}} \psi_{n_{r}}^{1 / \gamma}+n_{k} \mu<-b_{n_{r}} \psi_{n_{r}}^{1 / \gamma}\left(1-\mu / \psi_{n_{r}}^{1 /(\gamma-1)}\right) .
$$

The right-hand side factor in the above product of probabilities is thus less than

$$
\begin{aligned}
P\left(W_{n_{r}-n_{k}}\right. & \left.<-b_{n_{r}-n_{k}} \psi_{n_{r}}^{1 / \gamma}\left(1-\mu / \psi_{n_{r}}^{1 /(\gamma-1)}\right)\right) \\
& \sim C_{1}\left(\exp \left[-k_{\gamma} c_{1} \gamma / \gamma-1\right]\right) P\left(W_{n_{r}}<-b_{n_{r}} \psi_{n_{r}}^{1 / \gamma}\right)
\end{aligned}
$$

by Lemma $c^{\prime},(8)$ and (9). We consider next the case in which (62) does not hold, i.e.

$$
b_{n_{r}}<b_{n_{k}} \psi_{n_{r}}^{1 / \gamma(\gamma-1)}
$$

Let $Q_{k}$ denote the set of such indices $r$. We evaluate an upper bound for the number of indices contained in $Q_{k}$. From (55) we get the double inequality:

$$
b_{n_{k}}^{\gamma} \prod_{j=k}^{r}\left(1+a / \psi_{n_{j}}^{1 /(\gamma-1)}\right) \leqq b_{n_{r}}^{\gamma}<b_{n_{k}}^{\gamma} \prod_{j=k}^{r}\left(1+b / \psi_{n j}^{1 /(\gamma-1)}\right),
$$

and hence

$$
(r-k) \leqq(2 \gamma / a) \lg \left(b_{n_{r}} / b_{n_{k}}\right) .
$$

Applying (53) we find that the total number of indices in $Q_{k}$ does not exceed $C_{2}\left(\lg \lg b_{n_{k}}\right)^{1+\eta}$. We consider now the subset $Q_{k}^{1}$ of $Q_{k}$ for which:

$$
b_{n_{r}}>q b_{n_{k}}
$$

where $q=\exp [a(\gamma-1) / 4 \gamma b]$. By Lemma f we have:

$$
\begin{aligned}
P_{r}< & P\left(W_{n_{k}}<-b_{n_{k}} \psi_{n_{k}}^{1 / \gamma}\right) P\left(W_{n_{r}-n_{k}}<-b_{n_{r}} \psi_{n_{r}}^{1 / \gamma}\left(1-q^{-1 / 2}\right)\right) \\
& +P\left(W_{n_{k}}<-b_{n_{r}} \psi_{n_{r}}^{1 / \gamma} q^{-1 / 2}\right)=\mathrm{I}+\mathrm{II} .
\end{aligned}
$$

Now,

$$
\mathrm{I}<P\left(W_{n_{k}}<-b_{n_{k}} \psi_{n_{k}}^{1 / \gamma}\right) \exp \left[-k_{\gamma} \psi_{n_{r}}^{1 /(\gamma-1)}\left(1-q^{-1 / 2}\right)^{\gamma /(\gamma-1)}\right]
$$

whereas

$$
\begin{aligned}
\mathrm{II} & <P\left(W_{n_{k}}<-b_{n_{k}}\left(b_{n_{r}} / b_{n_{k}}\right) \psi_{n_{r}}^{1 / \gamma} q^{-1 / 2}\right) \\
& <P\left(W_{n_{k}}<-b_{n_{k}} \psi_{n_{r}}^{1 / \gamma} q^{1 / 2}\right) \\
& <C_{3} \psi_{n_{r}}^{-1 / 2(\gamma-1)} \exp \left[-k_{\gamma} \psi_{n_{r}}^{1 /(\gamma-1)} q^{\gamma / 2(\gamma-1)}\right] \\
& <C_{3} \psi_{n_{k}}^{-1 / 2(\gamma-1)}\left\{\exp \left[-k_{\gamma} \psi_{n_{k}}^{1 /(\gamma-1)}\right]\right\}\left\{\exp \left[-k_{\gamma} \psi_{n_{r}}^{1 /(\gamma-1)}\left(q^{\gamma / 2(\gamma-1)}-1\right)\right]\right\} \\
& =C_{4} P\left(W_{n_{k}}<-b_{n_{k}} \psi_{n_{k}}^{1 / \gamma}\right) \exp \left[-k_{\gamma} \psi_{n_{r}}^{1 /(\gamma-1)}\left(q^{\gamma / 2(\gamma-1)}-1\right)\right] .
\end{aligned}
$$

(53), (68), and (69) combine to give: 


$$
\begin{aligned}
\sum_{r \in Q_{k^{1}}} P_{r} & <2 C_{4} P\left(W_{n_{k}}<-b_{n_{k}} \psi_{n_{k}}^{1 / \gamma}\right) \sum_{r \in Q_{k}^{1}}\left(\lg b_{n_{r}}\right)^{-p_{\gamma}} \\
& <2 C_{5} P\left(W_{n_{k}}<-b_{n_{k}} \psi_{n_{k}}^{1 / \gamma}\right)\left(\lg \lg b_{n_{k}}\right)^{1+\eta}\left(\lg b_{n_{k}}\right)^{-p_{\gamma}} \\
& <M_{1} P\left(W_{n_{k}}<-b_{n_{k}} \psi_{n_{k}}^{1 / \gamma}\right)
\end{aligned}
$$

where $p_{\gamma}=\min \left\{\left(1-q^{-1 / 2}\right)^{\gamma /(\gamma-1)} / 2,\left(q^{\gamma / 2(\gamma-1)}-1\right) / 2\right\}$ and use is made of the considerations in the paragraph following (66). The rest of our argument is now nearly the same as in the case $0<\gamma<1$. We refer back to those pages for the detailed steps of the proof. We have yet to consider the set $Q_{k}^{2}$ of for which $b_{n_{r}}<q b_{n_{k}}$. For such $r$ it follows from (66) that:

$$
(r-k)<\psi\left(q b_{n_{k}}\right)^{1 /(\gamma-1)}(\gamma-1) / 4 b<\psi_{n_{k}}^{1 /(\gamma-1)} / 2 b .
$$

We can then expand both sides of the inequalities (65) to obtain:

$$
b_{n_{k}}\left[a(\gamma+1)(r-k) / \gamma^{2} \psi_{n_{r}}^{1 /(\gamma-1)}\right]<b_{n_{r}}-b_{n_{k}}<b_{n_{k}} 2^{1 / \gamma+1} b(r-k) / \gamma \psi_{n_{k}}^{1 /(\gamma-1)}
$$

and

$$
n_{r}-n_{k}<2^{1 / \gamma+1} b(r-k) n_{k} / \psi_{n_{k}}^{1 /(\gamma-1)}
$$

hence

$$
b_{n_{r}-n_{k}}<C_{6}(r-k)^{1 / \gamma} b_{n_{k}} / \psi_{n_{k}}^{1 / \gamma(\gamma-1)}
$$

We again let $A$ be a constant less than $b$; for $r \in Q_{k}^{2}$ we break up the probability $P_{r}$ into two parts. The first part is less than

$$
P_{r}^{\prime}=P\left(W_{n_{k}}<-b_{n_{k}} \psi_{n_{k}}^{1 / \gamma}\left(1+(r-k) A / \psi_{n_{k}}^{1 /(\gamma-1)}\right)\right)
$$

and hence

$$
\begin{aligned}
\sum_{r \in Q_{2}^{k}} P_{r}^{\prime} & <C_{7} \exp \left[-k_{\gamma} c_{2}(r-k)\right] P\left(W_{n_{k}}<-b_{n_{k}} \psi_{n_{k}}^{1 / \gamma}\right) \\
& <M_{2} P\left(W_{n_{k}}<-b_{n_{k}} \psi_{n_{k}}^{1 / \gamma}\right) .
\end{aligned}
$$

The remainder of $P_{r}$ is by Lemma $f$ inferior to:

$$
\begin{aligned}
\sum_{r \in Q_{2}^{k}} P\left(W_{n_{k}}<-b_{n_{k}} \psi_{n_{k}}^{1 / \gamma}\right) P\left(W_{n_{r}-n_{k}}<-b_{n_{r}} \psi_{n_{r}}^{1 / \gamma}+b_{n_{k}} \psi_{n_{k}}^{1 / \gamma} .\right. \\
\left.\quad \cdot\left(1+(r-k) A / \psi_{n_{k}}^{1 /(\gamma-1)}\right)\right) \\
<P\left(W_{n_{k}}<-b_{n_{k}} \psi_{n_{k}}^{1 / \gamma}\right) \sum_{r \in Q_{2}^{k}} P\left(W_{n_{r}-n_{k}}\right. \\
\left.<-b_{n_{r}-n_{k}}\left(b_{n_{k}} / b_{n_{r}-n_{k}}\right) c_{3}(r-k) / \psi_{n_{k}}^{1 / \gamma(\gamma-1)}\right) \\
<P\left(W_{n_{k}}<-b_{n_{k}} \psi_{n_{k}}^{1 / \gamma}\right) \sum_{r \in Q_{2}^{k}} P\left(W_{n_{r}-n_{k}}<-c_{4} b_{n_{r}-n_{k}}(r-k)^{(\gamma-1) / \gamma}\right),
\end{aligned}
$$


where $c_{2}, c_{3}$, and $c_{4}$ are positive, by (72) and (73). Hence

$$
(74)<M_{3} P\left(W_{n_{k}}<-b_{n_{k}} \psi_{n_{k}}^{1 / \gamma}\right) \text {. }
$$

(63), (70), (74), and (75) together verify conditions $c$ of Chung-Erdös.

4. Strong upper bounds for $S_{n}$ and $W_{n}$.

Theorem III. Let $\psi(n) \uparrow \infty, \psi(n) / n \downarrow 0$. Then

$$
\begin{array}{ll}
0<\gamma<1 ; & P\left(S_{n}>b_{n} / \psi\left(b_{n}\right)^{1 / \gamma} \text { i.o. }\right)=0 \text { or } 1, \\
1<\gamma<2 ; & P\left(W_{n}>b_{n} \psi\left(b_{n}\right)^{1 / \gamma} \text { i.o. }\right)=0 \text { or } 1
\end{array}
$$

as

$$
I_{3}(\psi)=\int_{1}^{\infty} \frac{1}{x \psi(x)} d x<\infty \text { or }=\infty .
$$

(This condition is equivalent to: $\sum_{n} \psi\left(2^{n}\right)<\infty$ or $=\infty$.)

5. Application to recurrent events. Let now the r.v. $X_{1}$ be the first recurrence time of a recurrent event $\varepsilon$. Let $N_{n}$ be the number of occurrences of $\varepsilon$ in the first $n$ trials. The following inverse relations hold between $N_{n}$ and $S_{n}$ :

$$
\begin{aligned}
& N_{n}>m \leftrightarrows S_{m}<n, \\
& N_{n}<m \rightleftarrows S_{m}>n .
\end{aligned}
$$

Theorems I and II can then be reformulated as follows:

Theorem I': Let $0<\gamma<1$, let $\phi(n) \uparrow \infty$, then

$$
P\left(N_{n} \geqq n^{\gamma} \phi\left(b_{n}\right) / h(n) \text { i.o. }\right)=0 \text { or } 1
$$

as $I_{1}(\phi)<\infty$ or $=\infty$.

TheOREM II': Let $1<\gamma<2$, let $\phi(n) \uparrow \infty$, then

$$
P\left(N_{n} \geqq(n / \mu)-\left(n^{1 / \gamma} / \mu^{(\gamma+1) / \gamma}\right) \phi\left(b_{n}\right) \text { i.o. }\right)=0 \text { or } 1 \text {, }
$$

as $I_{2}(\phi)<\infty$ or $=\infty$.

These theorems give an answer to the problem raised by Feller [3, p. 115] in the case where the function $h(x)$ satisfies the restrictions stated in the introduction of this paper.

\section{BIBLIOGRAPHY}

1. W. Doeblin, Sur l'ensemble des puissances d'une loi de probabilité, Studia Mathematica vol. 9 (1940) p. 71.

2. M. Lipschutz, On the magnitude of the error in the approach to stable distributions, available in mimeographed form from the Department of Mathematical Statistics, Columbia University. To be submitted for publication.

3. W. Feller, Fluctuation theory of recurrent events, Trans. Amer. Math. Soc. vol. 67 (1949) p. 98. 
4. - A limit theorem for random variables with infinite moments, Amer. J. Math. vol. 68 (1946) p. 257.

5. J. Marcinkiewicz, Quelques thêorèmes de la théorie des probabilités, Bulletin du Séminaire Mathématique de l'Université Wilno vol. 2 (1939) p. 22.

6. P. Lévy, Sur les séries dont les termes sont des variables éventuelles indépendantes, Studia Mathematica vol. 3 (1931) p. 117.

7. K. L. Chung and G. A. Hunt, On the zeros of $\Sigma \pm 1$, Ann. of Math. vol. 50 (1949) p. 383.

8. M. Lipschutz, On strong laws for certain types of events connected with sums of independent random variables, Ann. of Math. vol. 57 (1953) p. 318.

9. W. Feller, The general form of the so-called law of the iterated logarithm, Trans. Amer. Math. Soc. vol. 54 (1943) p. 373.

10. K. L. Chung and P. Erdös, On the applications of the Borel-Cantelli lemma, Trans. Amer. Math. Soc. vol. 72 (1952) p. 179.

UNIVERSITY OF MARYLAND, College Park, Md.

Columbia University, NEW YoRK, N. Y. 